\title{
A possible new dipping X-ray source in the field of M 31
}

\author{
V. Mangano ${ }^{1}$, G. L. Israel ${ }^{1}$, and L. Stella ${ }^{1}$ \\ Osservatorio Astronomico di Roma, Via Frascati 33, 00040 Monteporzio Catone (Roma), Italy \\ Received 5 August 2003 / Accepted 14 January 2004
}

\begin{abstract}
We report the discovery of the new dipping X-ray source, XMMU J004308.6+411247, in M 31, during a systematic search for periodicities in XMM-Newton archival observations. During the 2002 January 6 observation, the dips recur with a 107 min period and the source count rate is consistent with zero at the dip minimum. Dips with the same modulation and period are also observed during the XMM-Newton observations carried out on 2000 June 25, 2001 June 29 and the Chandra observation of 2001 October 5. The dips of XMMU J004308.6+411247 show no evidence of energy dependence. The average X-ray flux of XMMU J004308.6+411247 is nearly constant across different observations $\left(\sim 10^{37} \mathrm{erg} \mathrm{s}^{-1}\right.$ for an assumed M 31 distance of $780 \mathrm{kpc}$ in the $0.3-10 \mathrm{keV}$ band); the spectrum is well fitted by an absorbed power law with a photon index $\sim 0.8$ or an absorbed Comptonization model. The photo-electric absorption is consistent with the Galactic value in the source direction. If XMMU J004308.6+411247 is located in M 31 its properties are consistent with those of dipping low mass X-ray binaries in the Galaxy. Present observations do not make it possible to distinguish between dips and eclipses.

The possibility that XMMU J004308.6+411247 is a foreground X-ray source cannot be ruled out at present; in this case the source might be a magnetic cataclysmic variable.
\end{abstract}

Key words. galaxies: individual: M 31 - X rays: stars - binaries: close

\section{Introduction}

Being the closest large spiral (Sb) galaxy to our own, the Andromeda galaxy (M31) provides us with a prime opportunity to study X-ray emission in a galaxy similar to the Milky Way both in morphology and size. The sources in M31 are observed at the nearly uniform and well known distance of $\sim 780 \mathrm{kpc}$ (Stanek \& Garnavich 1998; Macri et al. 2001). Moreover, owing to its inclination angle of $\sim 77^{\circ}$ and high Galactic latitude ( $l \mathrm{II}=121.174312, b \mathrm{II}=-21.573022)$ sources are viewed through a substantially lower absorption column $\left(N_{\mathrm{H}} \sim 7 \times 10^{20} \mathrm{~cm}^{2}\right.$, Dickey \& Lockman 1990) than for sources in the Galaxy. Because of this moderate and fairly uniform extinction, M 31 sources can be studied and compared over an extended low energy band. Moreover, different stellar populations in the bulge, spiral arms and halo can be easily distinguished based on their location.

The XMM-Newton mission (Jansen et al. 2001) yields an unprecedented opportunity to study the time variability and spectral properties of individual X-ray sources in nearby galaxies with increased throughput. Previous observations of M 31 performed with Einstein (Trinchieri \& Fabbiano 1991; van Speybroeck et al. 1979) and ROSAT (Primini et al. 1993) revealed many point-like X-ray sources in its core $\left(r<5^{\prime} \sim\right.$ $1.1 \mathrm{kpc}$ ), but were too insensitive for their precise characterization. The two deep and extensive ROSAT-PSPC surveys of

Send offprint requests to: V. Mangano, e-mail: mangano@mporzio.astro.it the entire M31 disk (Supper et al. 2001, 1997), though effective in mapping the Globular Cluster (GC) and Supernova Remnant (SNR) populations, proved inadequate for a detailed study of point sources in the core of the galaxy because of the limited spatial resolution. Spatial resolution was the main limitation also in the study of the bulge of M 31 carried out with Beppo-SAX, the first to image M 31 up to energies of $\sim 10 \mathrm{keV}$ (Trinchieri et al. 1999).

The observations of the central part of M31 with the Chandra X-ray Observatory (Weisskopf 1988) resolved the nuclear source seen with the Einstein and ROSAT-HRI into five point sources (Garcia et al. 2000) and detected and localized bulge point sources down to luminosities of $\sim 2 \times$ $10^{35} \mathrm{erg} \mathrm{s}^{-1}$ in the $0.3-7 \mathrm{keV}$ band, with positional errors of less than 1" (Kong et al. 2002; Di Stefano et al. 2002; Kaaret 2002). None of these observations, however, was long enough for a detailed timing analysis. The large collecting area and bandpass of XMM-Newton afforded a more accurate characterization of both the global properties of the X-ray emission from M31 (Shirey et al. 2001) and individual X-ray sources in its bulge (Osborne et al. 2001; Barnard et al. 2002; Trudolyubov et al. 2002). M31 was selected as an XMM-Newton Performance Verification target (Jansen et al. 2001) and subsequently observed within the Guaranteed Time Program. The XMM-Newton observations of M31 (see Sect. 2) are among the M31 X-ray observations with the longest total exposure; they are eminently suited for timing and spectral analysis. We started a program of systematic search 
Table 1. XMM-Newton observations of M 31 used in this analysis.

\begin{tabular}{cccccc}
\hline \hline Observation & $\begin{array}{c}\text { Date } \\
(\mathrm{UT})\end{array}$ & $\begin{array}{c}T_{\text {start }} \\
(\mathrm{UT})\end{array}$ & $\begin{array}{c}T_{\text {stop }} \\
(\mathrm{UT})\end{array}$ & $\begin{array}{c}\text { Exposure time } \text { MOS }^{a} \\
(\mathrm{ks})\end{array}$ & $\begin{array}{c}{\text { Exposure time } \mathrm{PN}^{a}} \\
(\mathrm{ks})\end{array}$ \\
\hline 1 & $25 / 06 / 2000$ & $08: 12: 41$ & $20: 59: 33$ & $34.8(30.1)$ & $31.0(26.5)$ \\
2 & $28 / 12 / 2000$ & $00: 01: 33$ & $03: 43: 23$ & $12.2(12.2)$ & $9.9(9.9)$ \\
3 & $29 / 06 / 2001$ & $06: 15: 17$ & $22: 20: 20$ & $54.9(31.0)$ & $52.4(31.0)$ \\
4 & $06 / 01 / 2002$ & $18: 00: 56$ & $11: 52: 53^{b}$ & $63.0(63.0)$ & $60.1(60.1)$ \\
\hline
\end{tabular}

${ }^{a}$ Effective exposures used in our analysis. The number in parentheses is the exposure after exclusion of time intervals with high background levels.

${ }^{b}$ The following day.

for periodicities, with Fourier techniques, among all bright $\left(\gtrsim 5 \times 10^{35} \mathrm{erg} \mathrm{s}^{-1}\right)$ point-like X-ray sources detected in the XMM-Newton archival observations of M 31. Our search strategy is aimed at detecting periodic signals over the widest possible period range (see Sect. 3).

In this paper we report the discovery of a $107 \mathrm{~min}$ diplike modulation (nearly $100 \%$ amplitude) in the 2002 January 6 light curve of the source XMMU J004308.6+411247. The same modulation is clearly seen also in other XMM-Newton observations of M31 and in the ACIS-S Chandra observation of 2001 October 5 (see Sect. 4.1).

In the following sections we describe the set of data we analysed (Sect. 2), summarise the periodicity search technique (Sect. 3) and illustrate the properties of the dip we discovered in the light curve of XMMU J004308.6+411247 (Sect. 4). Our results are discussed in Sect. 5.

Throughout the paper we adopt a distance to M31 of $780 \mathrm{kpc}$ (Stanek \& Garnavich 1998; Macri et al. 2001) and a Galactic column density in the direction of M31 of $7 \times$ $10^{20} \mathrm{~cm}^{-2}$ (Dickey \& Lockman 1990).

\section{Observations and data analysis}

We used the four archival XMM-Newton observations of the core of M 31 listed in Table 1. The 2000 June 25 observation (observation 1 in Table 1) was part of the Performance Verification Program (Jansen et al. 2001; Shirey et al. 2001; Osborne et al. 2001). The other three observations (observations $2,3,4$ ) were carried out on 2000 December 28, 2001 June 29 and 2002 January 6 respectively as a part of Guaranteed Time Program. We used data from the three European Photon Imaging Camera (EPIC) instruments at the focus of the three co-aligned $1500 \mathrm{~cm}^{2} \mathrm{X}$-ray telescopes on board XMM-Newton: two EPIC MOS detectors (Turner et al. 2001) and one EPIC PN detector (Strüder et al. 2001). Each MOS detector is sensitive in the $0.2-10 \mathrm{keV}$ band, while the PN detector is sensitive in the $0.1-15 \mathrm{keV}$ band. In all observations, the three quoted detectors were operated in the full window mode $\left(\sim 30^{\prime}\right.$ diameter field of view) with medium (observations 1, 2 and 3) and thin (observation 4) optical blocking filters. We reduced the EPIC data with the XMM-Newton Science Analysis System (SAS) version 5.4.1, by performing standard screening of the EPIC data in order to exclude time intervals with high background levels and/or solar flares. This

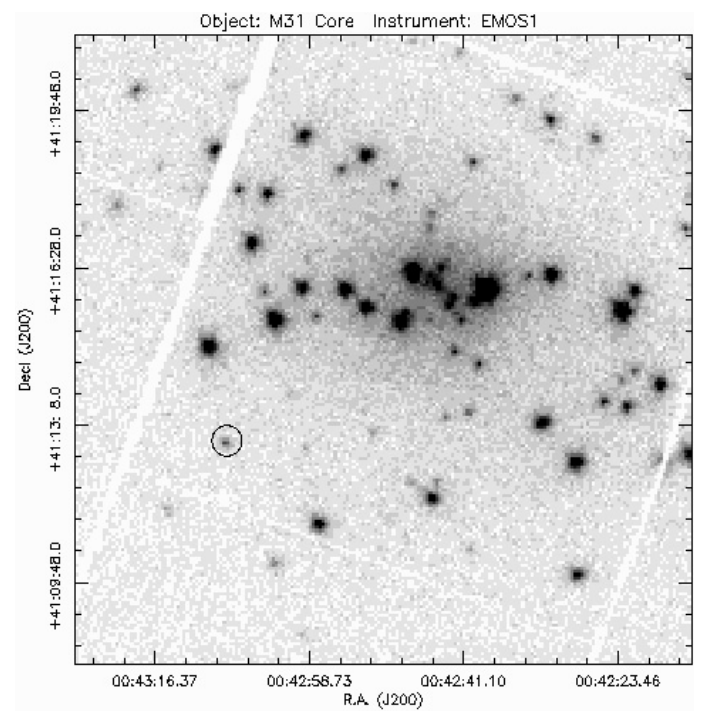

Fig. 1. Image of M31 from the XMM-Newton observation carried out on 2002 January 6 (MOS1 data). The source XMMU J004308.6+411247 is marked by the extraction circle that we used, containing $98 \%$ of the source counts.

resulted in shortened exposures of observations 1 and 3 as indicated in Table 1. We applied the automatic source detection and analysis pipeline summarised in the following section to all four observations. Observations 1, 3 and 4 have high enough statistics to carry out a detailed timing analysis of more than half of the individual point sources in them. Observation 2 has a somewhat lower exposure. Thus, Sect. 4 present only the results we obtained from observations 1, 3 and 4. In Fig. 1 the central part of the MOS1 image of M 31 extracted from the 2002 January 6 observation is shown.

\section{Search strategy}

For each observation listed in Table 1 we filtered PN, MOS1 and MOS2 events in the energy band $0.2-15 \mathrm{keV}$, and merged the events of the two MOS detectors into a single MOS1+MOS2 event list (task merge). We then produced images of the PN and MOS1+MOS2 fields binned in square pixels of $2^{\prime \prime} \times 2^{\prime \prime}$. Note that this is smaller than the FWHM of the point spread function in the center of the field for both PN and MOS, which is $\sim 6^{\prime \prime}$ for the PN and $\sim 4^{\prime \prime}$ for the MOS (XMM-Newton Users' Handbook V2.1 2003). We detected 
all sources above the $5 \sigma$ threshold in the two images (task ewavelet). For a description of the wavelet-based source detection algorithm used by the task ewavelet see Damiani et al. 1997. For every detected source this task yields the source position and extent, corresponding to the standard deviation of an equivalent Gaussian luminosity profile, i.e. the radius containing $\sim 66 \%$ of the source counts. In the longest exposure observation (observation 4 ) we found $\sim 350$ sources in the PN image and $\sim 180$ in the MOS image. We removed from the source lists some spurious sources close to the image edge, CCD borders and bad pixel lines (this was important especially in PN images).

After this we extracted light curves of all detected point sources and applied the barycentric correction to the photon arrival times. We used an extraction radius of two times the source extent calculated by ewavelet (i.e. $2 \sigma$ of the equivalent Gaussian luminosity profile; this contains about $98 \%$ of the source counts) whenever there was no superposition with nearby sources, and an extraction radius of one time the source extent in all other cases.

Finally we considered all extracted light curves containing more than 200 photons (including background) in order to have enough statistics for timing analysis. In this way, for instance, we reduced the total number of analysed sources to $\sim 140$ (PN) and $\sim 90$ (MOS) in observation 4. A detailed discussion on the complete set of sources that we analysed will be presented in a forthcoming paper.

The light curves obtained were searched for pulsations by using the power spectrum technique developed by Israel \& Stella (1996). To maximize signal detection sensitivity we calculated a single power spectrum with the highest Fourier resolution $\Delta v=1 / \Delta T$ (with $\Delta T$ the time span covered by the observation). In each power spectrum we searched for peaks above the detection threshold up to the Nyquist frequency $v_{\mathrm{Nyq}}=1 / 2 \Delta t$, with the binning time $\Delta t$ equal to the intrinsic time resolution of the EPIC cameras (i.e. $73.4 \mathrm{~ms}$ for the PN and $2.6 \mathrm{~s}$ for the MOS). The detection threshold for peaks in a single power spectrum containing $N_{\text {trial }}=\Delta T / 2 \Delta t$ frequencies was set such that the probability of exceeding it by chance in a total number of trials equal to $N_{\text {trial }}$ times the number $n$ of light curves analysed in the image was $\epsilon=n * N_{\text {trial }} * \epsilon_{\text {single trial }}$, with $\epsilon=2.326 \times 10^{-4}$ (corresponding to $3.5 \sigma$ in a Gaussian approximation). Thus, the detection threshold for the analysis of a single power spectrum was given by $\epsilon / n$. In this way, the minimum detectable amplitude (or pulsed fraction) for a sinusoidal signal (i.e. the sensitivity of the search to coherent periodic signals) for each source can be derived according to the prescription given by Vaughan et al. (1994).

\section{Results}

\subsection{Timing analysis}

The systematic search described in Sect. 3 revealed both the $865 \mathrm{~s}$ pulsation of the transient super-soft source XMMU J004319.4+411759 (Osborne et al. 2001) visible in the 2000 June 25 observation (observation 1) and quiescent in all other archival XMM-Newton observations, and the

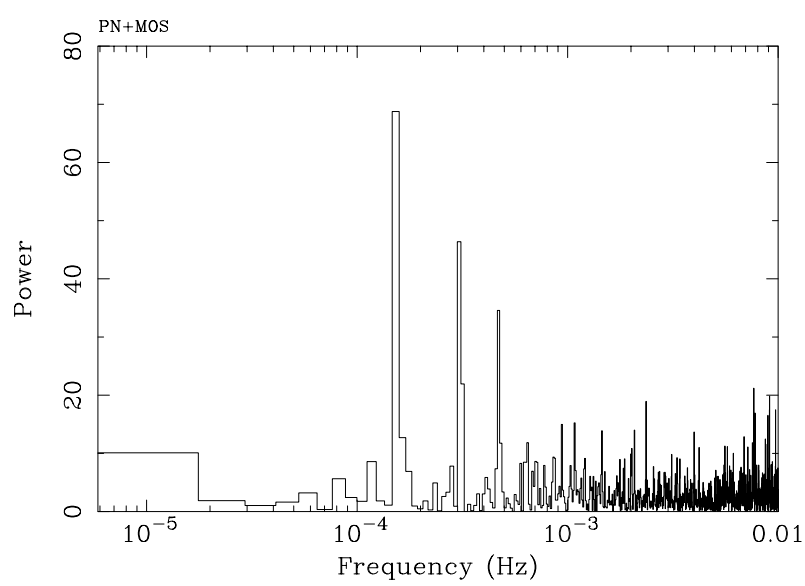

Fig. 2. Power spectrum of the combined PN and MOS light curves of the source XMMU J004308.6+411247 corresponding to the 2002 January 6 observation. The power spectrum is normalized following Leahy et al. (1983).

$2.78 \mathrm{~h}$ dips in the 2002 January 6 observation (observation 4) of XMMU J004314.1+410724 in the globular cluster Bo 158 (Trudolyubov et al. 2002).

We also found a significant peak at $1.525879 \times 10^{-2} \mathrm{~Hz}$ in the power spectrum of XMMU J004308.6+411247 extracted from observation 4 MOS data. The source position we obtained, RA $=00^{\mathrm{h}} 43^{\mathrm{m}} 08^{\mathrm{s}} .611 \mathrm{Dec}=41^{\circ} 12^{\prime} 47^{\prime \prime} .58$ (equinox 2000), should be considered accurate to better than $4^{\prime \prime}$. The power spectrum peak has a significance of $\sim 4.3 \sigma$ after taking into account the number of trial frequencies for this source, as well as for the other sources in the MOS image of observation 4 for which the periodicity search could be carried out. Visual inspection of the power spectra of the 2002 January 6 MOS and PN light curves of XMMU J004308.6+411247 clearly reveals the presence of the second and the third harmonics of the signal. As a further confirmation, we calculated the power spectrum of the combined PN and MOS light curves (using the XRONOS package, version 5.19) and obtained a significant increase in the power of the signal and its harmonics. In Fig. 2 the power spectrum of the PN+MOS light curve of XMMU J004308.6+411247 is shown. The signal fundamental plus two harmonics can be easily seen. An epoch folding search was carried out on the PN, MOS and PN+MOS light curves of XMMU J004308.6+411247 from 2002 January 6 observation aiming at a more accurate determination of the period. Following the prescription of Leahy (1987) we obtained an average best period of $6420 \pm 80 \mathrm{~s}$. For the background count rate estimation we used an annular region around the extraction circle of XMMU J004308.6+411247 (see also Sect. 4.2). The background-subtracted $\mathrm{PN}+\mathrm{MOS}$ light curve folded at a period of $6420 \mathrm{~s}$ is shown in Fig. 3 (panel a). The reference epoch $T_{0}$ corresponding to the minimum of the modulation is given in Table 2, together with the source count rate. The minima are consistent with a zero count rate. The dips last $\sim 25 \%$ of each cycle and their fall and rise are fairly continuous. A fit of the folded light curve in Fig. 3 with a simple model consisting of a constant and a Gaussian with negative normalization centered at the minimum gives a nearly $100 \%$ amplitude of the dip with 
Table 2. Parameters of the observed modulation.

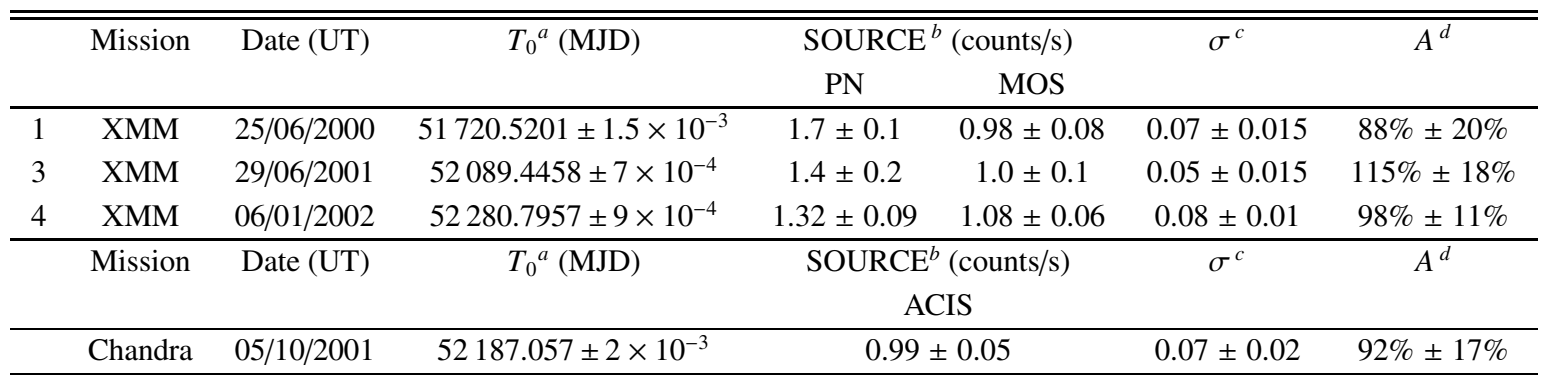

${ }^{a}$ Reference epoch corresponding to the minimum of the modulation.

${ }^{b}$ Source count rate in units of $10^{-2}$ counts/s in the $0.2-15 \mathrm{keV}$ band for the XMM-Newton observations and in the $0.3-10 \mathrm{keV}$ band for the Chandra observation. The MOS column refers to the MOS1+MOS2 data.

${ }^{c}$ Standard deviation of the Gaussian dip in phase units.

Errors are at the $90 \%$ confidence level. ${ }^{d}$ Amplitude of the modulation with respect to the out-of-dip intensity. Errors are at the $90 \%$ confidence level.

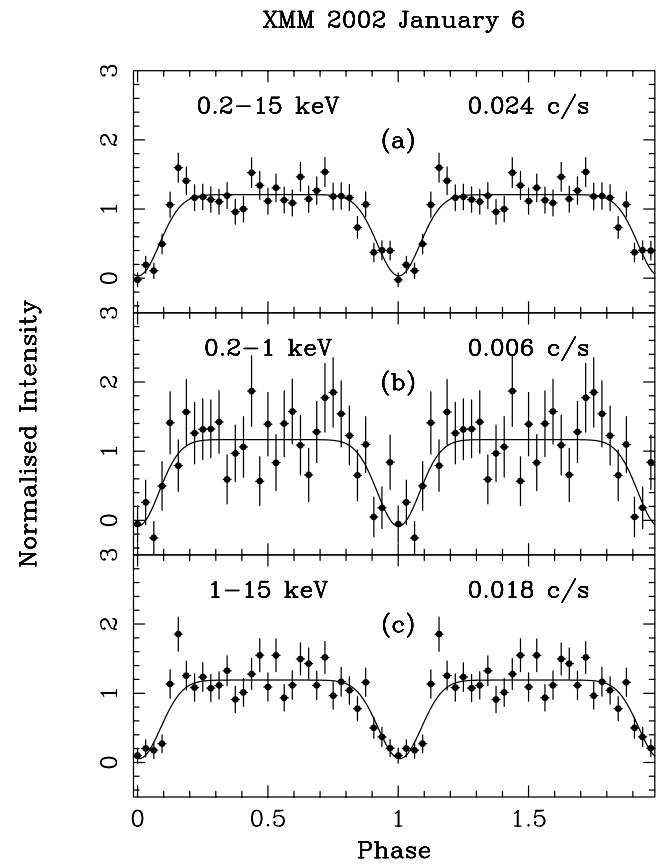

Fig. 3. Panel a) shows the combined PN+MOS light curve of XMMU J004308.6+411247 from the XMM-Newton observation of 2002 January 6 (observation 4), in the energy band $0.2-15 \mathrm{keV}$, and folded at the best period of $6420 \mathrm{~s}$. The solid line represents the fit of the data with the model described in the text (a constant minus a Gaussian). Panels b) and c) show the results obtained by folding at $6420 \mathrm{~s}$ the combined PN+MOS light curve in the $0.2-1 \mathrm{keV}$ and in the $1-15 \mathrm{keV}$ energy band, respectively. Each light curve is backgroundsubtracted and normalized to the average source count rate value given in the top right corner of the panel.

respect to the out-of-dip intensity and a $\sigma=(8.3 \pm 1.2) \times 10^{-2}$ (phase units). See Table 2. The same results are obtained from the combined $\mathrm{PN}+\mathrm{MOS}$ light curve restricted to the $0.2-1 \mathrm{keV}$ and $1-15 \mathrm{keV}$ energy bands and folded at the $6420 \mathrm{~s}$ period (see Fig. 3, panels b and c). The fit to the folded light curve gives a dip amplitude of $104 \% \pm 27 \%$ and $95 \% \pm 12 \%$ in the $0.2-1 \mathrm{keV}$ and in the $1-15 \mathrm{keV}$ energy band respectively, fully compatible with the $98 \% \pm 11 \%$ amplitude obtained in the $0.2-15 \mathrm{keV}$ energy band. We thus conclude that the data provide no evidence for an energy dependence of the modulation.

We searched for a modulation at a period around $6420 \mathrm{~s}$ also in all the other XMM-Newton observations of XMMU J004308.6+411247, using combined PN+MOS light curves of the source in all cases in order to improve statistics. An epoch-folding search yields a best period of $6400 \pm 440 \mathrm{~s}$ in observation 1 and $6425 \pm 200 \mathrm{~s}$ in observation 3 . The errors are a factor 3-5 larger than in observation 4 and the best period values are consistent with one another. Therefore we are justified in adopting the same period of $6420 \mathrm{~s}$ to fold all the light curves. We folded the XMMU J004308.6+411247 light curves extracted from XMM-Newton observations 1 and 3 at the $6420 \mathrm{~s}$ period and fitted the folded light curve with the same model used for observation 4 (a constant and a Gaussian with negative normalization). We confirmed the presence of the signal at the same period and with a modulation amplitude compatible with that previously found. Results are shown in Fig. 4 (panels a and b) and summarised in Table 2.

To investigate XMMU J004308.6+411247 further we used archival Chandra and ROSAT observations of M31. We selected the archival Chandra observations with the longest exposure, i.e. that carried out on 2001 October 5 with the Imaging Spectrometer ACIS-S (Garmire et al. 1992; Bautz 1998 and references therein) for an effective exposure of $\sim 37.7 \mathrm{ks}$, and the 2001 November 1 observation with the HRC-I in imaging mode for an effective exposure of $\sim 46.8 \mathrm{ks}$ (Murray et al. 1997). We used standard event processing and filtering procedures in the CIAO package, Version 2.2.1, and extracted light curves of XMMU J004308.6+411247 from circular regions of diameter about twice the FWHM of the local Point Spread Function (PSF), in the $0.3-10 \mathrm{keV}$ band. We folded the XMMU J004308.6+411247 light curves obtained from Chandra data at the $6420 \mathrm{~s}$ period. The dips are clearly detected in the ACIS-S Chandra observation carried out on 2001 October 5 (see panel c in Fig. 4) and their properties are consistent with those of the XMM-Newton observations (see Table 2). The best period obtained with an epoch-folding search on the ACIS-S Chandra observation of 2001 October 5 


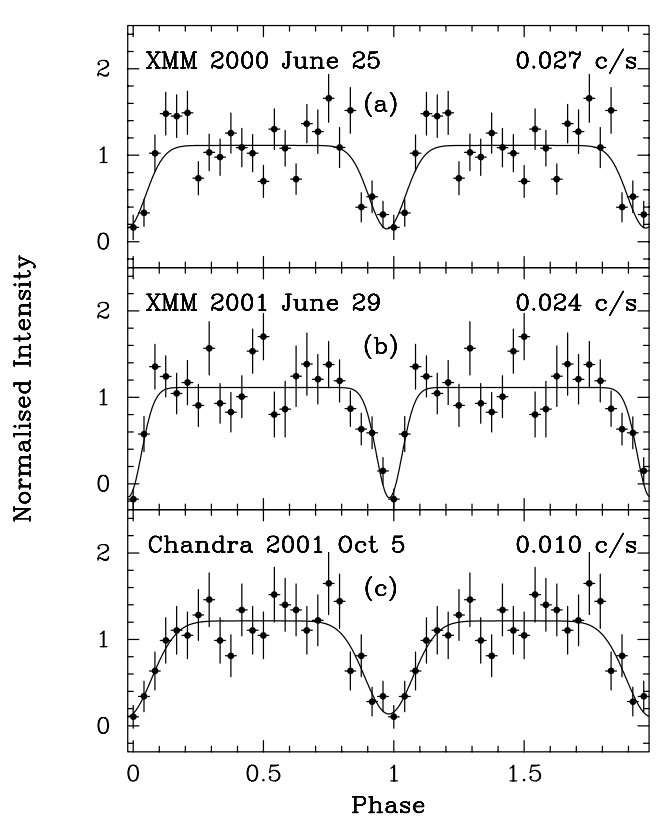

Fig. 4. We show the combined $\mathrm{PN}+\mathrm{MOS}$ light curves of XMMU J004308.6+411247 in the band $0.2-15 \mathrm{keV}$ folded at 107 min period extracted from XMM-Newton observations of 2000 June 25 a) and 2001 June 29 b). The fit with a simple model consisting of a constant and a Gaussian with negative normalization is also shown (solid line). In panel c) we show the folded light curve of an ACIS-S Chandra observation of the source of 2001 October 5. Each light curve is background subtracted and normalized to the source average count rate. This is given in the top right corner of each panel.

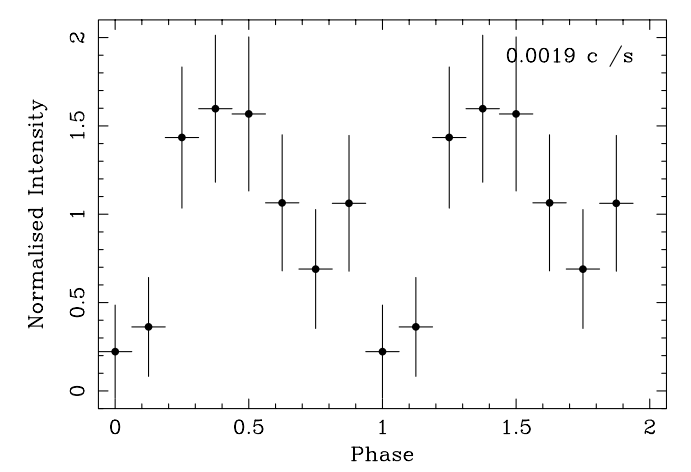

Fig. 5. Background-subtracted and folded light curve of XMMU J004308.6+411247 from the Chandra HRC-I observation of 2001 November 1. The average source count rate is given in the top right corner of the panel.

is $6437 \pm 200 \mathrm{~s}$, consistent with the period during observation 4. There is marginal evidence for the $107 \mathrm{~min}$ signal also in the HRC-I Chandra observation of 2001 November 1 (see Fig. 5) though the epoch-folding search does not show any significant peak.

We then considered the longest exposure ROSAT archival observations of M 31 containing XMMU J004308.6+411247 within ten arc-minutes from the center of the field of view. These are the 1996 January 7 HRI observation (85.5 ks exposure) and the 1991 July 27 PSPC observation (30 ks exposure). The ROSAT light curves of XMMU J004308.6+411247 contain

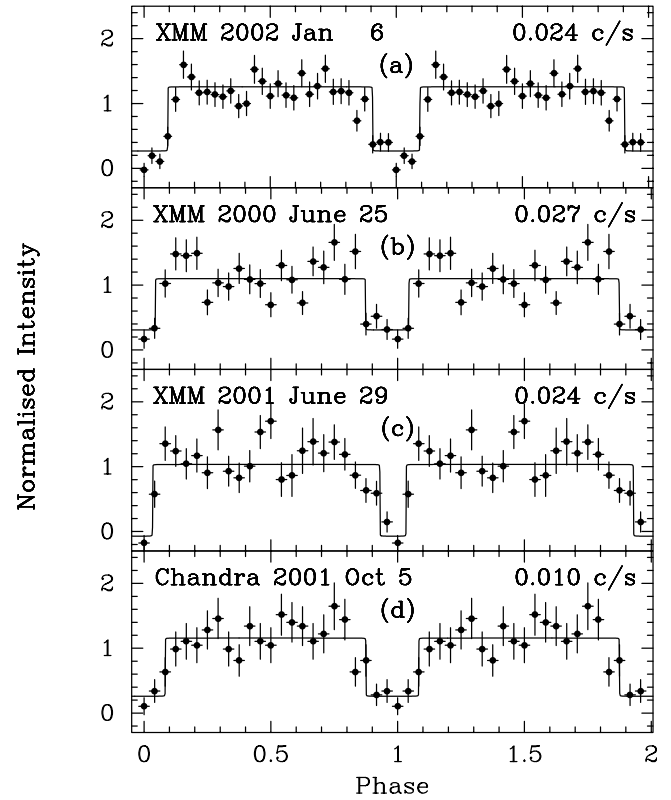

Fig. 6. Square-wave fit of the background-subtracted and folded light curves of XMMU J004308.6+411247 from the XMM-Newton and Chandra observations showing the 107 min pulsation. The satellite, observation date and average source count rate are given in each panel. The data are the same as in Fig. 3 panel a) and in Fig. 4 panels a)-c).

too few photons ( $\sim 60$ and $\sim 80$ net source counts respectively) to confirm the presence of the dips.

Note that the four folded light curves of XMMU J004308.6+411247 obtained from the XMM-Newton and Chandra data can be reasonably well fitted also by a square wave. For this we adopted a model of the form $Y(F)=D\left[\arctan \left(A\left(F_{1}-F\right)\right) / \pi+\arctan \left(A\left(F-F_{2}\right)\right) / \pi\right]+C$, where $A, F_{1}, F_{2}, D$ and $C$ are the parameters of the model and $F$ represent the phase. For very large values of $A\left(F_{2}-F_{1}\right)$ $(>1000)$ this expression produces an eclipse-type behaviour with sharp ingress and egress at phases $F_{1}$ and $F_{2}$, respectively. When $A\left(F_{2}-F_{1}\right)$ is smaller the ingress and egress become progressively smoother, $F_{1}$ and $F_{2}$ being the half depth phases. When $A\left(F_{2}-F_{1}\right) \lesssim 100$ the profile turns into a dip-like one with no flat minimum phase interval.

We fit all our folded light curves to the square-wave model with $A$ frozen to $10^{4}$, the $\chi^{2}$ being insensitive to changes in $A$ within one order of magnitude from this value. The results are shown in Fig. 6. The square-wave model fit provides a reduced $\chi^{2}$ slightly closer to 1 than the Gaussian fit, but uncertainties in the parameters D and C (needed for the eclipse depth estimation) are large. According to the square-wave model fit the XMM-Newton 2002 January 6 light curve profile shows an eclipse lasting $0.19 \pm 0.03$ phase units with a $92 \% \pm 38 \%$ decrease of the source flux during the eclipse $(90 \%$ confidence level errors). In the XMM-Newton 2000 June 25 light curve the eclipse lasts $0.21 \pm 0.06$ phase units and produces an $84 \% \pm 45 \%$ flux decrease; in the XMM-Newton 2001 June 29 it lasts $0.125 \pm 0.005$ and gives a $104 \% \pm 60 \%$ flux decrease and finally, in the Chandra 2001 October 5 observation 
the eclipse lasts $0.21 \pm 0.03$ and gives an $89 \% \pm 49 \%$ flux decrease.

Because of these results we conclude that poor statistics does not allow us to distinguish between a smooth profile of the folded light curve (like the one represented by the Gaussian model) or a sharp eclipse-like light curve profile.

\subsection{Spectral analysis}

We extracted phase-averaged spectra of XMMU J004308.6+411247 in the $0.2-15 \mathrm{keV}$ energy band from the PN and MOS data of all the XMM-Newton observations in which the $107 \mathrm{~min}$ modulation was detected (observations 1,3 and 4). We generated the relevant spectral response matrices (SAS tasks rmfgen and arfgen). The background spectrum was extracted from the same annular region around the source extraction circle used for the background count rate estimation. These spectra were analysed simultaneously by fitting the following spectral models: absorbed power law, absorbed power law and blackbody, absorbed power law with exponential cut off, absorbed thermal bremsstrahlung, absorbed Comptonization model (Titarchuk 1994; Titarchuk \& Lyubarskij 1995) with spherical or disk geometry. Given the relatively poor statistics of our spectra, it is perhaps not surprising that a simple absorbed power law provides a statistically acceptable fit (see below). Nonetheless we fit also a larger sample of two components spectral models that are often used to describe the spectra of various classes of Galactic pulsating $\mathrm{X}$-ray sources in order to try to draw some closer analogies. The spectral fitting was performed using the same model parameters for the PN, MOS1 and MOS2 phase-averaged spectra. We allowed for independent normalizations of the three spectra in order to account for calibration differences between the EPIC cameras. Results from the spectral fitting are given in Table 3. Only models yielding a reduced $\chi^{2}$ less than 2 are reported. The model consisting of an absorbed power law with an exponential cut off was rejected because it yields a cutoff energy greater than $100 \mathrm{keV}$, i.e. a spectrum virtually indistinguishable from a power law in the XMM-Newton energy band. Note that the absorbed Comptonization model (Titarchuk 1994) gives an acceptable fit only by assuming a disk geometry for the Comptonising region.

The model consisting of a simple absorbed power law gives the best reduced $\chi^{2}$ in all observations, but provides an upper limit to the absorption column at $95 \%$ confidence level that is lower than the Galactic value of $7 \times 10^{20} \mathrm{~cm}^{-2}$ derived from the HI distribution map by Dickey \& Lockman 1990. The latter has a very limited angular resolution $\left(1^{\circ} \times 1^{\circ}\right)$. It was estimated that small scale structure in the HI distribution in the sky introduces errors in the column density estimates of $\pm 1 \times 10^{20} \mathrm{~cm}^{-2}$ ( $90 \%$ confidence level, see Elvis et al. 1986, Appendix B). The upper limits to $N_{\mathrm{H}}$ derived from the absorbed power-law fit are below the $90 \%$ confidence interval for the Galactic value. The fit with the Comptonization model has the same problem in two out of three observations. The inclusion of a black body component in the absorbed power law model (this is done in analogy with the X-ray spectra modeling used for several

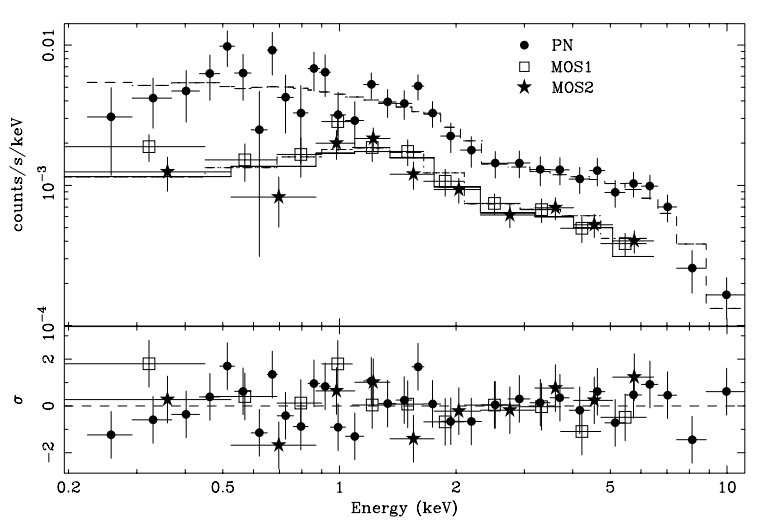

Fig. 7. Phase-averaged spectra of XMMU J004308.6+411247 extracted from PN, MOS1 and MOS2 data of the XMM-Newton observation carried out on 2002 January 6 . The resulting simultaneous fit with the absorbed power law model is shown (see Table 3). The dashed line gives the best fit model for the PN spectrum, the dot-dashed is for the MOS1 and the solid line for the MOS2.

Galactic dipping sources, see e.g. XB 1916-053, Church et al. 1997; XB 1254-690, Smale et al. 2002; X 1624-49, Church \& Bałucińska-Church 1995) gives plausible upper limits to the absorption column, but is not required statistically since the power-law fit is already acceptable. The phase-averaged spectra of XMMU J004308.6+411247 obtained from the 2002 January 6 observation are shown in Fig. 7.

To investigate further the low-level absorption indicated by the above fits, we also fit groups of PN, MOS1 and MOS2 phase-averaged spectra with the three models discussed above, while freezing $N_{\mathrm{H}}$ at the Galactic value (which is to be considered a lower limit to the absorption for a source located in M31). We obtain reduced $\chi^{2}$ values of 1.2 (power law), 1.03 (power law plus blackbody) and 1.1 (comptonization) for observation 1, of 1.4, 0.96 and 1.2 for observation 3 and of 1.3, 0.76 and 1.06 for observation 4 respectively and parameter values consistent with the corresponding ones listed in Table 3 to within the errors. Note also that the upper limits to $N_{\mathrm{H}}$ increase considerably (up to values consistent with the Galactic value in some cases) when allowing for independent absorption columns in the PN, MOS1 and MOS2 spectra. The 2002 January 6 PN, MOS1 and MOS2 phase-averaged spectra fitted in this way with the absorbed power-law model gives a $\chi^{2}$ of 48.1 (47 d.o.f.), a photon index of $0.79_{-0.09}^{+0.1}$ and $N_{\mathrm{H}}<4 \times 10^{20} \mathrm{~cm}^{-2}$ for the PN spectrum, $N_{\mathrm{H}}<6.8 \times$ $10^{20} \mathrm{~cm}^{-2}$ for the MOS1 spectrum, $N_{\mathrm{H}}<1.4 \times 10^{20} \mathrm{~cm}^{-2}$ for the MOS2 spectrum. Fitting again the three spectra with the absorbed Comptonization model (disk geometry), we obtain a $\chi^{2}$ of 37.20 (45 d.o.f.), an input photon temperature of $k T_{0}<0.12 \mathrm{keV}$, Comptonising electron temperature of $k T_{\mathrm{e}}=3.3_{-0.8}^{+2.6} \mathrm{keV}$, and optical depth $\tau=12.5_{-1.75}^{+1.8}$ and $N_{\mathrm{H}}<$ $6.8 \times 10^{20} \mathrm{~cm}^{-2}$ for the PN spectrum, $N_{\mathrm{H}}<11.5 \times 10^{20} \mathrm{~cm}^{-2}$ for the MOS1 spectrum, $N_{\mathrm{H}}<1.7 \times 10^{20} \mathrm{~cm}^{-2}$ for the MOS2 spectrum. Based on this analysis we conclude that the low $95 \%$ confidence upper limits to $N_{\mathrm{H}}$ obtained may be related to calibration uncertainties of the EPIC cameras. It is worth noting that XMMU J004308.6+411247 is not the only source we found showing this anomalous behaviour. Also the bright 
Table 3. Best-fit model parameters of energy spectra of XMMU J004308.6+411247 ${ }^{a}$

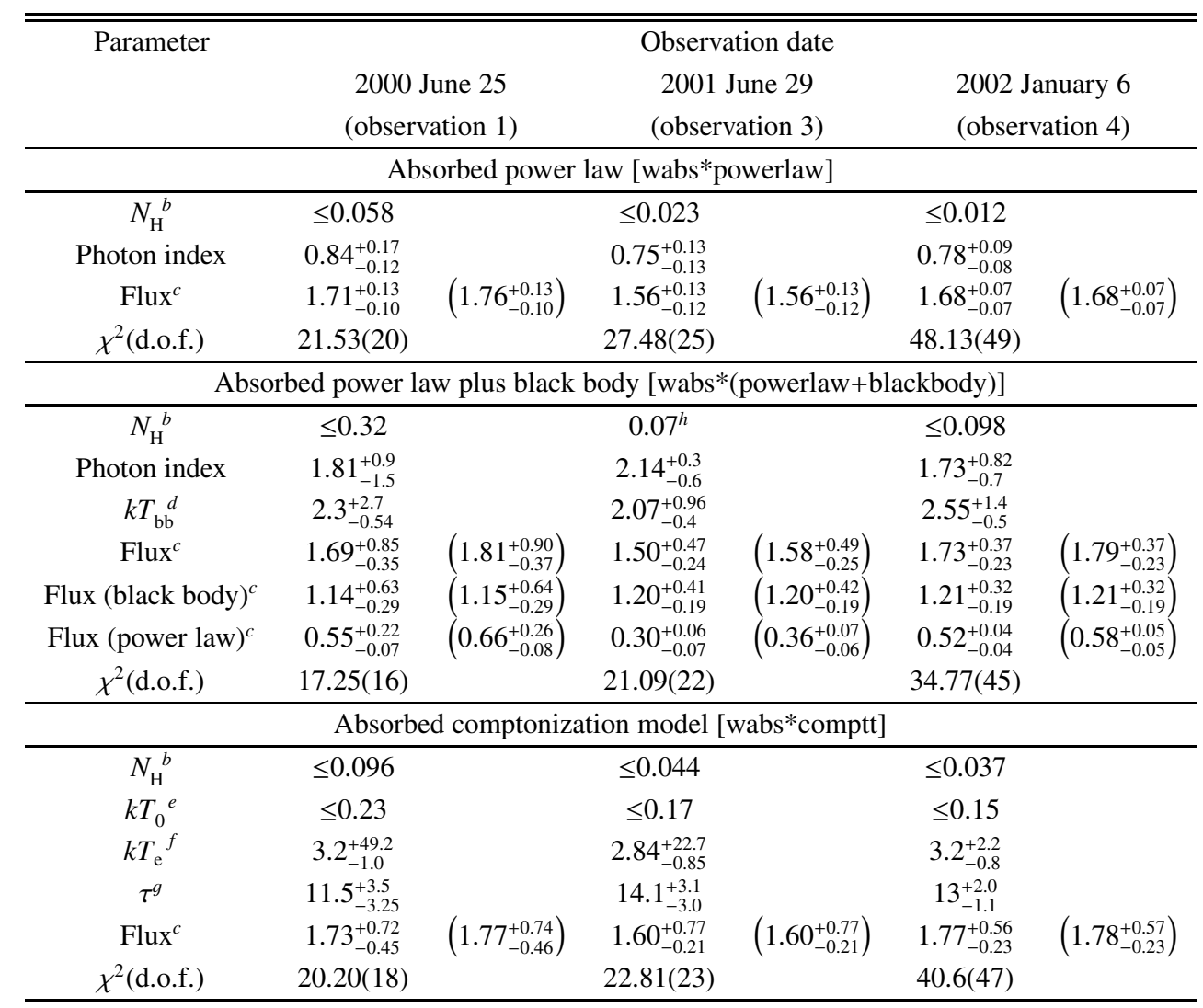

${ }^{a}$ Results of simultaneous fit of EPIC-PN, MOS1 and MOS2 phase-averaged spectra in the $0.2-15 \mathrm{keV}$ energy band. Parameter errors correspond to $90 \%$ confidence level.

${ }^{b}$ Upper limit at $95 \%$ confidence level to the equivalent absorbing hydrogen column density in units of $10^{22} \mathrm{~cm}^{-2}$.

${ }^{c}$ Observed flux in the $0.3-10 \mathrm{keV}$ energy range in units of $10^{-13} \mathrm{erg} \mathrm{s}^{-1} \mathrm{~cm}^{-2}$. Average value from PN, MOS1 and MOS2. The value of the unabsorbed flux is in parentheses. Errors are calculated from the uncertainty in the normalization at the $67 \%$ confidence level.

${ }^{d}$ Blackbody temperature in $\mathrm{keV}$.

${ }^{e}$ Upper limit to the temperature of soft photons in keV.

${ }^{f}$ Electron temperature in $\mathrm{keV}$.

${ }^{g}$ Thomson optical depth for the disk geometry.

${ }^{h}$ Parameter frozen to the Galactic value.

source XMMU J004232.0+411314 shows spectra with typical photon index $\sim 1$ and $N_{\mathrm{H}}<0.6 \times 10^{20} \mathrm{~cm}^{2}$ at $95 \%$ confidence level in the 2002 January 6 observation. In this case the upper limit to $N_{\mathrm{H}}$ grows to $0.9 \times 10^{20} \mathrm{~cm}^{2}$ and to $1.2 \times 10^{20}$ in MOS1 and MOS2 spectra respectively when allowing for independent $N_{\mathrm{H}}$ parameters in the fit (we did not extract PN spectra because in the PN image the source is more than $50 \%$ covered by CCD edges and hot pixel lines). XMMU J004232.0+411314 corresponds to source r2-32 in the ACIS-I Chandra catalogue by Kong et al. (2002). In the quoted paper (Table 8) the source was listed among the 20 brightest sources in M 31 and its spectrum was well represented by a power-law spectral fit in the $0.5-10 \mathrm{keV}$ band with photon index $1.3_{-0.1}^{+0.2}$ and $N_{\mathrm{H}}<$ $5 \times 10^{20} \mathrm{~cm}^{2}$ at the $95 \%$ confidence level, i.e. an upper limit to the absorption column lower than the Galactic value.

Based on the absorbed flux values in the $0.3-10 \mathrm{keV}$ band given in Table 3, we can conclude that the luminosity of XMMU J004308.6+411247 was roughly constant over the $\sim 1.5 \mathrm{yr}$ time span covered by XMM-Newton observations, at a level of $\sim 1 \times 10^{37}\left(d / d_{\mathrm{M} 31}\right)^{2} \mathrm{erg} \mathrm{s}^{-1}$, where $d$ is the actual distance of XMMU J004308.6+411247 from us and $d_{\mathrm{M} 31}$ the M31 distance.

To investigate changes in the energy spectrum during the dips, we divided the PN, MOS1 and MOS2 events into two phase intervals corresponding to the out-of-dip (from phase 0.1 to phase 0.85 ) and dip intensity (from phase 0.85 to 0.1 ). We extracted spectra from events in the two quoted phase intervals, thus obtaining out-of-dip and dip spectra. The MOS1 and MOS2 dip spectra of observations 1 and 3 had less than 30 counts each (including background) and therefore were not used in any spectral fit. For each observation we fit the PN outof-dip and dip spectra simultaneously with the phase-averaged spectrum, using two different models: an absorbed power law and an absorbed Comptonization model. Independent normalizations and absorption columns for the three spectra were used, while the other parameters were frozen to the best fit values in Table 3. To within the obvious statistical limitations this approach is justified by the fact that simultaneous fits of the PN, 
Table 4. Best-fit model parameters of energy spectra of XMMU J004308.6+411247 ${ }^{a}$.

\begin{tabular}{|c|c|c|c|c|c|c|}
\hline \multirow[t]{4}{*}{ Parameter } & \multicolumn{6}{|c|}{ Observation date } \\
\hline & \multirow{3}{*}{\multicolumn{2}{|c|}{$\begin{array}{c}2000 \text { June } 25 \\
\text { (observation 1) } \\
\text { PN data }\end{array}$}} & \multirow{3}{*}{\multicolumn{2}{|c|}{$\begin{array}{c}2001 \text { June } 29 \\
\text { (observation 3) } \\
\text { PN data }\end{array}$}} & \multirow{2}{*}{\multicolumn{2}{|c|}{$\begin{array}{l}2002 \text { January } 6 \\
\text { (observation 4) }\end{array}$}} \\
\hline & & & & & & \\
\hline & & & & & \multicolumn{2}{|c|}{ PN, MOS1 and MOS2 data } \\
\hline \multicolumn{7}{|c|}{ Absorbed power law [wabs*powerlaw] } \\
\hline$N_{\mathrm{H}}(\text { out-of-dip })^{b}$ & $\leq 0.023$ & & $\leq 0.037$ & & $\leq 0.01$ & \\
\hline$N_{\mathrm{H}}(\operatorname{dip})^{b}$ & $\leq 9.7$ & & $\leq 0.93$ & & $\leq 0.22$ & \\
\hline Flux (out-of-dip) ${ }^{c}$ & $2.19_{-0.13}^{+0.14}$ & $\left(2.19_{-0.13}^{+0.15}\right)$ & $1.89_{-0.14}^{+0.14}$ & $\left(1.89_{-0.14}^{+0.14}\right)$ & $2.02_{-0.07}^{+0.07}$ & $\left(2.02_{-0.07}^{+0.07}\right)$ \\
\hline Flux (dip) $)^{c}$ & $1.01_{-0.31}^{+0.87}$ & $\left(1.09_{-0.33}^{+0.94}\right)$ & $1.09_{-0.28}^{+0.31}$ & $\left(1.09_{-0.28}^{+0.31}\right)$ & $0.69_{-0.13}^{+0.16}$ & $\left(0.69_{-0.13}^{+0.16}\right)$ \\
\hline$\chi^{2}$ (d.o.f.) & $39.74(26)$ & & $27.48(24)$ & & $85.85(95)$ & \\
\hline \multicolumn{7}{|c|}{ Absorbed comptonization model [wabs*comptt] } \\
\hline$N_{\mathrm{H}}(\text { out-of-dip })^{b}$ & $\leq 0.044$ & & $\leq 0.048$ & & $\leq 0.03$ & \\
\hline$N_{\mathrm{H}}(\operatorname{dip})^{b}$ & $\leq 32$ & & $\leq 0.90$ & & $\leq 0.19$ & \\
\hline Flux (out-of-dip) ${ }^{c}$ & $2.14_{-0.16}^{+0.44}$ & $\left(2.14_{-0.16}^{+0.44}\right)$ & $1.93_{-0.18}^{+0.53}$ & $\left(1.93_{-0.18}^{+0.53}\right)$ & $2.11_{-0.12}^{+0.34}$ & $\left(2.13_{-0.12}^{+0.34}\right)$ \\
\hline Flux (dip) $)^{c}$ & $1.03_{-0.32}^{+0.84}$ & $\left(1.16_{-0.35}^{+0.91}\right)$ & $1.11_{-0.29}^{+1.22}$ & $\left(1.11_{-0.29}^{+1.22}\right)$ & $0.73_{-0.15}^{+0.21}$ & $\left(0.73_{-0.15}^{+0.21}\right)$ \\
\hline$\chi^{2}$ (d.o.f.) & $33.12(25)$ & & $22.81(23)$ & & $70.79(94)$ & \\
\hline
\end{tabular}

${ }^{a}$ Results of simultaneous fit of the out-of-dip, dip and phase-averaged spectra of source XMMU J004308.6+411247. Spectral parameters are frozen to the values indicated in Table 3.

${ }^{b}$ Upper limit at $95 \%$ confidence level to the equivalent absorbing hydrogen column density in units of $10^{22} \mathrm{~cm}^{-2}$.

${ }^{c}$ Model observed flux in the $0.3-10 \mathrm{keV}$ energy range in units of $10^{-13} \mathrm{erg} \mathrm{s}^{-1} \mathrm{~cm}^{-2}$. The value of the unabsorbed flux is in parentheses. Errors are calculated from the uncertainty in the normalization at the $67 \%$ confidence level.

MOS1 and MOS2 out-of-dip spectra alone give results consistent with those obtained for the phase-averaged spectra listed in Table 3. A similar check cannot be done with dip spectra alone because of poor statistics, and the usage of the same spectral model fitting out-of-dip and phase-averaged spectra represents the simplest assumption. Results are shown in Table 4. Note that for the 2002 January 6 observation also the MOS1 and MOS2 phase-averaged, out-of-dip and dip spectra were fitted simultaneously to the PN data. In this case we used nine independent normalizations for the nine spectra and three independent $N_{\mathrm{H}}$ parameters, one for the three (PN, MOS1 ans MOS2) phase-averaged spectra, one for the out-of-dip spectra and one for the dip spectra. The PN dip and out-of-dip spectra from the 2002 January 6 observation are shown in Fig. 8 together with their best fit power law model. The resulting 95\% confidence upper limit on the average increase in the (neutral) absorbing column density $N_{\mathrm{H}}$ during the dips was $9.7 \times 10^{22} \mathrm{~cm}^{-2}$ in observation $1,9 \times 10^{21} \mathrm{~cm}^{-2}$ in observation 3 and $2 \times 10^{21} \mathrm{~cm}^{-2}$ in observation 4. (The tighter limit obtained for observation 4 clearly reflects the longer exposure time in that observation). However, the above limits should be taken as indicative, since in order to increase the $\mathrm{S} / \mathrm{N}$ of the dip spectrum, it was integrated over the whole duration of the dips (as opposed to a shorter phase interval around the dip minimum).

\subsection{Other observations}

An X-ray source with a position consistent with that of XMMU J004308.6+411247 was detected with the ROSAT-HRI (source [PFJ93]74, Primini et al. 1993), ROSAT-PSPC (sources 2RXP J004308.5+411251 and 2RXP J004308.3+411249 in the

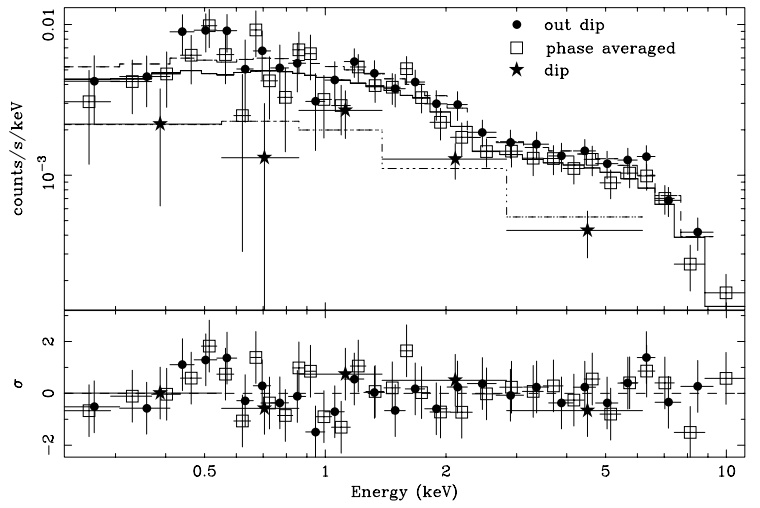

Fig. 8. out-of-dip, dip and phase-averaged spectra of XMMU J004308.6+411247 extracted from PN data of the XMM-Newton observation carried out on 2002 January 6. The resulting simultaneous best fit with the absorbed power law model with photon index $\Gamma=0.78$ is shown (see Tables 3 and 4). The dashed line gives the best fit model for the out-of-dip spectrum, the dot-dashed is for the dip spectrum and the solid line for the phase-averaged spectrum.

Second ROSAT PSPC Catalogue; source RX J0043.1+4112 in the M31 second ROSAT PSPC survey Supper et al. 2001), and Chandra (source CXOM 31 J004308.4+411247 in Kaaret 2002, source CXOM 31 J004308.6+411248 in Kong et al. 2002, source CXOM 31 J004308.6+411250 in Williams et al. 2003).

The observed flux of XMMU J004308.6+411247 from the latter experiments is roughly constant over a 10 years time span. From the published values of the source luminosity we derive values of the integrated unabsorbed flux 
in the $0.3-10 \mathrm{keV}$ band of $\sim 3.5 \times 10^{-14} \mathrm{erg} \mathrm{s}^{-1} \mathrm{~cm}^{-2}$ from ROSAT-HRI 1990 July 25 observation (Primini et al. 1993), $\sim 1.0 \times 10^{-13} \mathrm{erg} \mathrm{s}^{-1} \mathrm{~cm}^{-2}$ from Chandra-ACIS 1999-2000 observations (Kong et al. 2002), $\sim 8.5 \times 10^{-14} \mathrm{erg} \mathrm{s}^{-1} \mathrm{~cm}^{-2}$ from Chandra-HRC 1999-2001 observations (Williams et al. 2003), $\sim 3.4 \times 10^{-14} \mathrm{erg} \mathrm{s}^{-1} \mathrm{~cm}^{-2}$ from Chandra-HRC 2001 October 31 observation (Kaaret 2002). Note that both Primini et al. (1993) and Kaaret (2002) use a thermal bremsstrahlung distribution to fit the spectra, with temperature $k T=5 \mathrm{keV}$ and $2 \mathrm{keV}$ and absorption column $N_{\mathrm{H}}=7 \times 10^{20} \mathrm{~cm}^{-2}$ and $6.66 \times 10^{20} \mathrm{~cm}^{-2}$ respectively, while Kong et al. (2002) and Williams et al. (2003) use a power law with photon index $\Gamma=1.7$ and absorption column $N_{\mathrm{H}}=10^{21} \mathrm{~cm}^{-2}$. These values are within a factor five from the observed flux of $\sim 1.7 \times$ $10^{-13} \mathrm{erg} \mathrm{s}^{-1} \mathrm{~cm}^{-2}$ we obtain from XMM-Newton observations in the $0.3-10 \mathrm{keV}$ band (see Table 3 and Sect. 4.2). This indicates that XMMU J004308.6+411247 is a fairly stable X-ray source, and not an X-ray transient.

\section{Discussion}

The unprecedent throughput of XMM-Newton led to the detection of a 107 min modulation from the X-ray source XMMU J004308.6+411247 in the field of M31. This source is very likely the ROSAT-HRI source [PFJ93]74 (Primini et al. 1993). The modulation is confirmed also by Chandra archival observations of the source. The source flux was at a nearly steady level of $\sim 1.7 \times 10^{-13} \mathrm{erg} \mathrm{s}^{-1} \mathrm{~cm}^{-2}$ in the $0.3-10 \mathrm{keV}$ band throughout all XMM-Newton observations. This value is within a factor five from flux measurements obtained by other missions spanning the previous decade. The flux given above corresponds to a luminosity of $\sim 1 \times 10^{37}(d / 780 \mathrm{kpc})^{2} \mathrm{erg} \mathrm{s}^{-1}$, where $d$ is the distance of XMMU J004308.6+411247 from us. The source spectrum is well fitted by an absorbed power law model with a photon index $\Gamma \sim 0.8$, but is also compatible with an absorbed Comptonization model $\left(k T_{\mathrm{e}} \sim 3 \mathrm{keV}\right.$ and $\tau \sim 13)$. No other simple single-component spectral model succeeds in fitting the XMM-Newton data. Owing to poor statistics the $N_{\mathrm{H}}$ value could not be measured from the XMM-Newton spectra and only upper limits were derived.

The $107 \mathrm{~min}$ modulation is due to a dip in the light curve that covers about one fourth of the cycle with an energy-independent intensity decrease compatible with $100 \%$ at dip minimum. The apparent smoothness of the XMMU J004308.6+411247 light curves might be due to poor statistics. An eclipse-like behaviour is also compatible with the folded light curve of XMMU J004308.6+411247. In this case, however, the "eclipse" duration would be of 0.12 phase units during the XMM-Newton observation of 2001 June 29, inconsistent with the $\sim 0.2$ duration obtained for the other three observations. This indicates that the observed modulation might not be compatible with a true eclipse by a companion star.

The possibility that XMMU J004308.6+411247 is a foreground source in the Galaxy cannot be ruled out at present. Indeed the somewhat lower value of $N_{\mathrm{H}}$, compared to the galactic $N_{\mathrm{H}}$, that we inferred from the analysis of the X-ray spectrum would appear to favor this possibility.

\subsection{Galactic scenario}

For an assumed distance of $1 \mathrm{kpc}$, roughly corresponding to the thickness of the Galaxy in the direction of M31, the $0.3-10 \mathrm{keV}$ luminosity of XMMU J004308.6+411247 would be $\sim 2 \times 10^{31} \mathrm{erg} \mathrm{s}^{-1}$, close to the low end of (but still compatible with) the quiescent $\mathrm{X}$-ray luminosity of magnetic cataclysmic variables (CVs; Ramsay et al. 1994; Ramsay $\&$ Cropper 2004). The period of the X-ray modulation of XMMU J004308.6+411247 is suggestive of the orbital period of a polar or intermediate polar (IP). Orbital periods of polars and IPs are mostly concentrated in the $1-10 \mathrm{~h}$ range (Downes et al. 2001). Current limits on the magnitude of any optical counterpart to XMMU J004308.6+411247 ( $V>21.7$, $R>20.2$, see Kong et al. 2002; Haiman et al. 1994) are also compatible with a magnetic CV interpretation (see Downes et al. 2001).

Dip-like X-ray modulations and/or on/off shaped eclipses reflecting the orbital motion of the binary are seen in both polars and IPs. Examples of this are Fo Aqr, AO Psc (Hellier et al. 1993) and XY Arietis (showing eclipses, Hellier 1997) among IPs; 2A0311-227 (EF Eri, Beuermann et al. 1991), DP Leo (showing eclipses, Schwope et al. 2002) and, to lesser extent, AN UMa (Ramsay et al. 1994) among polars.

However, the longest duration of an eclipse obtained through occultation of a compact accreting X-ray source by a donor star filling its Roche lobe of radius $r_{\mathrm{L}}$ in a binary system with orbital period $P$, binary separation $a$ and mass ratio $q=M_{\text {donor }} / M_{\mathrm{X}}<1$ can be calculated as $\frac{1}{P} 2 r_{\mathrm{L}} / \frac{2 \pi a}{P}=\frac{r_{\mathrm{L}}}{\pi a}$ (in phase units). Using the relation of Eggleton (1983) to express the dependence of the Roche-lobe radius of the donor $r_{\mathrm{L}}$ on $q$ we derive an upper limit to an eclipse duration of $0.4 / \pi \sim 0.13$ phase units. A 0.2 phase units eclipse of the X-ray source cannot be due to occultation by the companion star. Thus, in any interacting binary interpretation, the observed X-ray modulation of XMMU J004308.6+411247 cannot be explained as due to a true eclipse.

If the dip ingress/egress in the XMMU J004308.6+411247 light curves were interpreted as sharp on the basis of the squarewave fit, a more plausible explanation for the observed modulation would be that of eclipses by the accretion column in a Polar. In this case no limit to the eclipse duration would be expected and occasional variations in the light curve profile may occur (see the case of EP Dra, Bridge et al. 2003).

The alternative scenario for XMMU J004308.6+411247 $\mathrm{X}$-ray modulation at the orbital period would be occultation of an IP by the plasma bulge due to the impact of the accretion stream on the accretion disk. Such a mechanism, at work both in IPs and low mass X-ray binaries (LMXRBs), might produce a relatively long dip which, when averaged, often has a fairly smooth profile, consistent with the Gaussian fit to the folded light curves that we described in Sect. 4.1. The apparent energy-independence of the dips (to within the uncertainties) might be accounted for if the plasma cloud producing the dips had a very high degree of ionization, and the flux reduction were due to Thomson scattering rather than photo-electric absorption. In the IP scenario the ionization front due to the central X-ray source cannot reach the edge of the accretion disk: to 
produce the $>90 \%$ energy-independent decrease of the source luminosity, an electron column $N_{\mathrm{e}}$ of more than $3.5 \times 10^{24} \mathrm{~cm}^{-2}$ would be required. To have this amount of ionized matter (with solar composition) in a cloud of thickness $\Delta R$ at a distance $R$ from the central source emitting an X-ray luminosity $L_{\mathrm{X}}$, the ionization parameter $\xi=\frac{L_{\mathrm{X}} \Delta R}{N_{\mathrm{e}} R^{2}}$ should be larger than 1000 (Tarter et al. 1969; Hatchett et al. 1976). We define $\epsilon=\frac{\Delta R}{R}$, i.e. the depth of the material that produces the dips expressed as a fraction of its distance from the source. We conservatively assume $\epsilon=1$. Thus, in the case of XMMU J004308.6+411247 the distance from the central X-ray source of the material causing the dips should be $R=\frac{L_{\mathrm{X}} \epsilon}{N_{\mathrm{e}} \xi}<10^{-3} \frac{L_{\mathrm{X}}}{N_{\mathrm{e}}}$. For an X-ray luminosity of $L_{\mathrm{X}} \sim 2 \times 10^{31} \mathrm{erg} \mathrm{s}^{-1}$ this maximum distance amounts to less than $1 \mathrm{~km}$, i.e. orders of magnitude lower than the radius of any bulge in the accretion stream/disk interaction.

Most IPs display soft X-ray modulation at the white dwarf (WD) spin period (33 s to $2 \mathrm{~h}$, but always shorter than the orbital period, Patterson 1994) with energy-dependent amplitudes in the 10-40\% range. No evidence was found for such a distinct X-ray modulation (possibly, in the soft X-ray range) in the case of XMMU J004308.6+411247. We note, however, that X-ray pulsations with amplitude $<30 \%$ would have remained undetected in the XMM-Newton light curves of XMMU J004308.6+411247.

A final possibility, producing a relatively smooth modulation in the X-ray light curve, is a self-eclipse of the hot emitting region on the polar cap of a highly magnetic WD (polar) in synchronous rotation with a low mass companion from which it accretes matter via Roche-lobe overflow without accretion disk formation. In this case the profile and duration of the eclipse would be due to the shape and extension of the hot polar cap periodically hidden at the WD spin period, equal to the orbital period. The energy independence of the eclipse depth would be also explained.

If XMMU J004308.6+411247 were a Galactic CV as suggested by its low X-ray luminosity for a $1 \mathrm{kpc}$ distance scale, the folded light curve modulation would be clearly more suggestive of an accretion stream eclipse or a self eclipse of the WD in a binary system of the polar class.

However, evidence against the magnetic CV interpretation derives from the analogy with the spectral characteristics observed from Polars and IPs. In both cases the observed spectra are compatible with a thermal bremsstrahlung spectrum with temperature in excess of tens of $\mathrm{keV}$, plus (in many cases) a very soft spectral component consistent with a blackbody of temperature in the $10-50 \mathrm{eV}$ range. The bolometric luminosity in this component is from one to several hundreds times higher than the thermal bremsstrahlung component in Polars (Ramsay \& Cropper 2004), while it ranges from zero (i.e. the component is not detected) to several tens of times the thermal bremsstrahlung component in IPs.

The spectral characteristics of XMMU J004308.6+411247 are at variance with those of magnetic CVs. The X-ray spectrum of XMMU J004308.6+411247 has a characteristic temperature $2-3 \mathrm{keV}$ (that of the Comptonising electrons) and, in any case, cannot be fit with a thermal bremsstrahlung spectrum. The addition of a soft blackbody component with temperature of $50 \mathrm{eV}$ or less produces a bolometric blackbody flux comparable to the bremsstrahlung, but the fit should be discarded because of reduced $\chi^{2}$ of 1.6 (in the best case).

Finally we note that, in the Galactic interpretation, the X-ray luminosity and spectrum of XMMU J004308.6+411247 would be similar to those observed from quiescent soft $\mathrm{X}$-ray transient low mass binaries hosting a neutron star (see e.g. Campana \& Stella 2000). X-ray eclipses have so far been observed only in a very low luminosity state of MXB 1659-29 (Wijnands et al. 2003); no evidence for a dip-like modulation of the X-ray flux at the orbital period has yet been found in any quiescent neutron star soft $\mathrm{X}$-ray transient. Therefore, while in principle viable, the possibility that XMMU J004308.6+411247 is a dipping quiescent soft X-ray transient is not especially appealing. On the other hand the interpretation of the X-ray modulation of XMMU J004308.6+411247 in terms of eclipses by the companion star is untenable because of the eclipse duration exceeds the maximum allowed duration (see above).

\subsection{Extragalactic scenario}

As an M31 source, the nearly steady luminosity of XMMU J004308.6+411247 ( 10 $\left.0^{37} \mathrm{erg} \mathrm{s}^{-1}, \quad 0.3-10 \mathrm{keV}\right)$ would be similar to that of moderately bright LMXRBs (White et al. 1988). The shape of the $107 \mathrm{~min}$ periodic modulation, comprising a fairly flat on-phase lasting for about $3 / 4$ of the cycle and a relatively long and smooth dip (1/4 of the cycle) reaching (nearly) zero flux over some $1 / 10$ of the cycle, is clearly reminiscent of X-ray dipping LMXRBs seen from high inclinations.

The possibility that the observed modulation is due to true eclipses of a compact central X-ray source by the companion star (supported by the square-wave fit of the folded XMMU J004308.6+411247 light curves shown at the end of Sect. 4.1), can be simply ruled out because the observed eclipse duration is incompatible with the geometry of the binary system (see Sect. 5.1).

LMXRB dips are believed to arise from absorption and/or Thomson scattering of X-rays coming from a central source by a bulge in the outer regions of the accretion disk, where the stream from the Roche-lobe filling companion impacts the outer disk rim. The dipping phenomenon reflects the orbital period of the system, as demonstrated by a few systems which display both X-ray dips and eclipses from the companion stars (e.g. MXB 1659-298, Cominsky \& Wood 1984, 1988; Wijnands et al. 2003; EXO 0748-676, Parmar et al. 1986; GRS 1747-312, in't Zand et al. 2000, 2003). Despite some jitter in phase and the common presence of pronounced dip variability, the position of the bulge must remain (nearly) fixed in phase (Frank et al. 1987). It is estimated that the dip phenomenon occurs in systems with an inclination greater than $\sim 70^{\circ}$. Some 20 dipping LMXRBs are currently known in the Galaxy. Only one of these (X 1916-053) has an orbital period shorter than $\sim 2 \mathrm{~h}$ (White \& Swank 1982), while two (4U 1755-338 and 4U 1630-47, in Mason et al. 1985 and Tomsik et al. 1998 respectively) likely host an accreting Black 
Hole Candidate (BHC). Most dipping LMXRBs are type I $\mathrm{X}$-ray bursters and thus contain an accreting neutron star.

In the dipping LMXRB interpretation XMMU J004308.6+411247 would be a compact binary, the second dipper with a period below the period gap.

The spectra of high luminosity $\left(>10^{37} \mathrm{erg} \mathrm{s}^{-1}\right.$ ) LMXRBs hosting a central neutron star are usually well fitted e.g. by a Comptonization model plus a blackbody component (White et al. 1988; for a review of other models see e.g. Di Salvo \& Stella 2002). When required, the blackbody component has a temperature around $1.5 \mathrm{keV}$ and it accounts for $\sim 10-30 \%$ of the total $1-10 \mathrm{keV}$ flux. For both low/high luminosity sources, Comptonization parameters are $y \sim 2-4$, optical depths $\tau \sim$ 10-15 and electron temperatures $T_{\mathrm{e}} \sim 2-4 \mathrm{keV}$. Spectra of (soft state) Black Hole Candidates in LMXRBs are well fitted by a Comptonization model too, but with a somewhat higher scattering depth $(\tau>15)$ and lower electron temperature $\left(T_{\mathrm{e}} \sim 1 \mathrm{keV}\right.$; White et al. 1988). LMXRBs with somewhat lower luminosity $\left(<10^{37} \mathrm{erg} \mathrm{s}^{-1}\right)$ are often dominated by a power law with photon index $\Gamma \gtrsim 2$ (Christian \& Swank 1997; Schulz 1999; Church \& Bałucińska-Church 2001).

In the case of XMMU J004308.6+411247, the XMM-Newton spectra are reasonably well fitted by a power law or a Comptonization model (see Table 3). The power-law fit gives a photon index $(\Gamma<1)$, i.e. harder than that found in LMXRBs. The addition of a blackbody component to the power law, though not required from the statistical point of view, implies too high a blackbody temperature $(\sim 2.3 \mathrm{keV})$ and fraction of the total luminosity $(\sim 70 \%)$ as compared to typical values for LMXRBs. On the contrary the thermal Comptonization model discussed in Sect. 4.2 yields an electron temperature of $\sim 3 \mathrm{keV}$ and an optical depth $\sim 13$ consistent with high luminosity LMXRBs hosting a neutron star.

In this interpretation other relevant features of XMMU J004308.6+411247 are:

(i) the $\sim 25 \%$ duty cycle of the dips; this is in agreement with those observed in Galactic dipping sources: the duty cycle of the dips varies from source to source and is typically $10 \%-40 \%$. Sources showing dips with a $20 \%$ duty cycle are, for example, 4U 1755-338 (Mason et al. 1985), XB 1254-690 (Courvoisier et al. 1986), MXB 1659-29 (Cominsky \& Wood 1984);

(ii) the shape of the dips, consistent with a relatively smooth average profile; this is common in Galactic dipping sources as well, despite the fact that individual dips often display pronounced irregular variability. Owing to poor statistics, individual dip variability would not be revealed in XMMU J004308.6+411247;

(iii) the very high degree of modulation, nearly $100 \%$; in turn this is not unusual in LMXRBs: e.g. 4U 1916-053 and XB 1254-690 often display more than 90\% modulated dips; MXB 1658-298 presented $~ 90 \%$ modulated dips in the Beppo-SAX observation of August 2000 (Oosterbroek et al. 2001); the dips of EXO 0748-676 occasionally reach a depth of $80 \%$ (Parmar et al. 1986);

(iv) the absence of a conspicuous increase in $N_{\mathrm{H}}$ during dips. The most constraining upper limit to the average absorption column of the obscuring bulge producing the dips we obtained from XMM-Newton observations of XMMU J004308.6+411247 is rather low: $<2 \times 10^{21} \mathrm{~cm}^{-2}$ (at $95 \%$ confidence level; see Table 4 ). However, we reiterate that the dip spectra were extracted over a fairly large phase interval (1/4 of a cycle), which samples predominantly the relatively shallow stages of the dip (this was done in order to increase the $\mathrm{S} / \mathrm{N}$ ). For this reason the upper limit we derived might underestimate the actual limit on the increase of $N_{\mathrm{H}}$ around dip minimum;

(v) the energy independence of the modulation (to within the uncertainties). We have already discussed this point in Sect. 5.1 (5th paragraph). As in the IP case, this may be accounted for if the disk bulge producing the dips were ionized by the central X-ray source and the flux reduction mechanism at work was Thomson scattering and not (as often happens) photo-electric absorption.

Following the same line of reasoning of Sect. 5.1 we conclude that for an X-ray luminosity $L_{\mathrm{X}} \sim 1 \times 10^{37} \mathrm{erg} \mathrm{s}^{-1}$ the distance from the central $\mathrm{X}$-ray source of the material causing the dips should be $R \lesssim 3 \times 10^{9} \mathrm{~cm}$, since only material closer than $3 \times$ $10^{9} \mathrm{~cm}$ from the X-ray source will be significantly ionized. By contrast, for a $107 \mathrm{~min}$ binary period the binary separation of the system is expected to be $a \sim 5.2 \times 10^{10} M_{\mathrm{X}}^{1 / 3}(1+q)^{1 / 3} \mathrm{~cm}$, with $M_{\mathrm{X}}$ the mass of the compact object in solar units, and $q$ the mass ratio of the donor star and the accreting star. Thus, the accretion disk is expected to have a radius of $\sim 0.3 a \lesssim 2 \times$ $10^{10} M_{\mathrm{X}}^{1 / 3} \mathrm{~cm}$ for stellar masses with ratio $q \lesssim 1$ (Whitehurst \& King 1991; Eggleton 1983). This requires, for ionization to be important, that the disk is at least one order of magnitude smaller than expected or that the material responsible for the dips is located further inside the edge of the disk, both of which seem unlikely. We note that in the case of 4U 1755-338 the ionization hypothesis also leads to the same difficulties (Mason et al. 1985).

Another possibility to interpret the energy-independent dips of XMMU J004308.6+411247 would be the eclipse by the companion star of an extended source of X-rays of comparable size, such as that originating from electron scattering in an Accretion Disk Corona (ADC). Besides the energyindependence, this would explain the smooth ingress and egress of the dips as well as the (nearly) $100 \%$ reduction of the observed X-ray flux. In this hypothesis, however, the central X-ray source should be completely hidden by the accretion disk rim to explain the absence of sharp eclipses. In analogy with known ADC sources we would thus expect that the true luminosity of XMMU J004308.6+411247 is one or two orders of magnitude higher than that observed, i.e. $10^{38}-10^{39} \mathrm{erg} \mathrm{s}^{-1}$. This in turn would suggest a $\mathrm{BHC}$ accreting at very high rates; however, the observed source spectrum is considerably harder than that expected from such a BHC.

\section{Conclusion}

We discovered $107 \mathrm{~min}$ periodic dips in the X-ray light curve of XMMU J004308.6+411247. The dip profile, amplitude, and average spectra of XMMU J004308.6+411247 
are consistent with a accreting neutron star in a short period LMXRB seen at a high inclination and located in M 31. The apparent energy-independence of the dips is more difficult to interpret in this scenario. If this interpretation is correct, XMMU J004308.6+411247 would be the second dipping source discovered in M 31 (after XMMU J004314.1+410724 in the globular cluster Bo 158, Trudolyubov et al. 2002).

The possibility that XMMU J004308.6+411247 is a foreground magnetic $\mathrm{CV}$ cannot be ruled out at present, but appears to be less likely.

Acknowledgements. We acknowledge a number of useful exchanges with F. Fiore, J. Osborne and A. Tiengo on the analysis of XMM-Newton data. The comments from an anonymous referee helped to improve the analysis and the presentation of the data.

This work was partially supported through ASI and MIUR-COFIN grants.

\section{References}

Barnard, R., Kolb, U., \& Osborne, J. P. 2002, Proc. Symp. New Visions of the X-ray Universe in the XMM-Newton and Chandra Era, ESA SP-488

Beuermann, K., Thomas, H.-C., \& Pietsch, W. 1991, A\&A, 246, L36

Bautz, M. W. 1998, Proc. SPIE, 3444, 210

Bridge, C. M., Cropper, M., Ramsay, G., et al. 2003, MNRAS, 341, 863

Campana, S., \& Stella, L. 2000, ApJ, 541, 849

Christian, D. J., \& Swank, J. H. 1997, ApJS, 109, 177

Church, M. J., \& Bałucińska-Church, M. 1995, A\&A, 300, 441

Church, M. J., \& Bałucińska-Church, M. 2001, A\&A, 369, 915

Church, M. J., Dotani, T., Bałucińska-Church, M., et al. 1997, ApJ, 491,388

Cominsky, L., \& Wood, K. 1984, ApJ, 283, 765

Cominsky, L., \& Wood, K. 1984, ApJ, 337, 485

Courvoisier, T. J.-L., Parmar, A. N., Peacock, A., \& Pakull, M. 1986, ApJ, 309, 265

Damiani, F., Maggio, A., Micela, G., \& Sciortino, S. 1997, ApJ, 483, 350

Dickey, J. M., \& Lockman, F. J. 1990, ARA\&A, 28, 215

Di Salvo, T., \& Stella, L. 2002, in The Gamma-Ray Universe, ed. A. Goldwurm, D. N. Neuman, \& J. Trân Thân Vân (Thê Gioi Publishers), Moriond Astrophys. Meet. Ser., 67

Di Stefano, R., Kong, A. K. H., Garcia, M. R., et al. 2002, ApJ, 570, 618

Downes, R. A., Webbink, R. F., Shara, M. M., et al. 2001, PASP, 113, 764, http://icarus.stsci.edu/downes/cvcat

Eggleton, P. P. 1983, ApJ, 268, 368

Elvis, M., Green, R. F., Bechtold, J., et al. 1986, ApJ, 310, 291

Frank, J., King, A. R., \& Lasota, J. P. 1987, A\&A, 178, 137

Garcia, M. R., Murray, S. S., Primini, F. A., et al. 2000, ApJ, 537, L23

Garmire, G. P., Ricker, G. R., Bautz, M. W., et al. 1992, Space Programs and Technologies Conference (New York: American Institute of Aeronautics \& Astronautics), 8

Haiman, Z., Magnier, E., Lewin, W. H. G., et al. 1994, A\&A, 286, 725 Hatchett, S., Buff, J., \& McCray, R. 1976, ApJ, 206, 847

Hellier, C. 1997, MNRAS, 291, 71

Hellier, C., Garlick, M. A., \& Mason, K. O. 1993, MNRAS, 260, 299

in't Zand, J. J. M., Bazzano, A., Cocchi, M., et al. 2000, A\&A, 355, 145 in't Zand, J. J. M., Hulleman, F., Markwardt, C. B., et al. 2003, A\&A, 406, 233

Israel, G. L., \& Stella, L. 1996, ApJ, 468, 369

Jansen, F., Lumb, D., Altieri, B., et al. 2001, A\&A, 365, L1

Kaaret, P. 2002, ApJ, 578, 114

Kong, A. K. H., Garcia, M. R., Primini, F. A., et al. 2002, ApJ, 577, 738

Leahy, D. A. 1983, ApJ, 266, 160

Leahy, D. A. 1987, A\&A, 180, 275

Macri, L. M., Calzetti, D., Freedman, W. L., et al. 2001, ApJ, 549, 721

Mason, K. O., Parmar, A. N., \& White, N. E. 1985, MNRAS, 216, 1033

Murray, S. S., Chappell, J. H., Kenter, A. T., et al. 1997, Proc. SPIE, 3114,11

Oosterbroek, T., Parmar, A. N., Sidoli, L., in’t Zand, J. J. M., \& Heise, J. 2001, A\&A, 376, 532

Osborne, J. P., Borozdin, K. N., Trudolyubov, S. P., et al. 2001, A\&A, 378,800

Parmar, A. N., White, N. E., Giommi, P., \& Gottwald, M. 1986, ApJ, 308, 199

Patterson, J. 1994, PASP, 106, 209

Primini, F. A., Forman, W., \& Jones, C. 1993, ApJ, 410, 615

Ramsay, G., Mason, K. O., Cropper, M., Watson, M. G., \& Clayton, K. L. 1994, MNRAS, 270, 692

Ramsay, G., \& Cropper, M. 2004, MNRAS, 347, 497

Schulz, N. S. 1999, ApJ, 511, 304

Schwope, A. D., Hambaryan, V., Schwarz, R., Kanbach, G., \& Gänsicke, B. T. 2002, A\&A, 392, 541

Shirey, R., Soria, R., Borozdin, K., et al. 2001, A\&A, 365, L195

Smale, A. P., Church, M. J., \& Bałucińska-Church, M. 2002, ApJ, 581, 1286

Stanek, K. Z., \& Garnavich, P. M. 1998, ApJ, 503, L131

Strüder, L., Briel, U., Dennerl, K., et al. . 2001, A\&A, 365, L18

Supper, R. 1997, A\&A, 317, 328

Supper, R. 2001, A\&A, 373, 63

Tarter, C. B., Tucker, W. H., \& Salpeter, E. E. 1969, ApJ, 156, 943

Titarchuk, L. 1994, ApJ, 434, 570

Titarchuk, L., \& Lyubarskij, Y. 1995, ApJ, 450, 876

Tomsik, J. A., Lapshow, I., \& Kaaret, P. 1998, ApJ, 494, 747

Trinchieri, G., Israel, G. L., Chiappetti, L., et al. 1999, A\&A, 348, 43

Trudolyubov, S. P., Borozdin, K. N., Priedhorsky, W. C., et al. 2002, ApJ, 581, L27

Trinchieri, G., \& Fabbiano, G. 1991, ApJ, 382, 82

Turner, M. J. L., Abbey, A., Arnaud, M., et al. 2001, A\&A, 365, L27

van Speybroeck, L., Epstein, A., Forman, W., et al. 1979, ApJ, 234, L45

Vaughan, B. A., van der Klis, M., Wood, K. S., et al. 1994, ApJ, 435, 362

Weisskopf, M. C. 1988, Space Sci. Rev., 47, 47

White, N. E., Stella, L., \& Parmar, A. N. 1988, ApJ, 324, 363

White, N. E., \& Swank, J. H. 1982, ApJ, 253, L61

Whitehurst, R., \& King, A. 1991, MNRAS, 249, 25

Williams, B. F., Garcia, M. R., Kong, A. H. K., et al. 2003, preprint [arXiv: astro-ph/0306421]

Wijnands, R., Nowak, M., Miller, J. M., et al. 2003, ApJ, 594, 952

XMM-Newton Users' Handbook V2.1 2003, ed. M. Ehle, M. Breitfellner, R. Gonzales Riestra, et al., http://xmm.vilspa.esa.es/external/xmm_user_support/ documentation/uhb/XMM_UHB.html 